\title{
Harmonic Domain Characterization of the Resonant Interaction Between Generator and Transmission Line
}

\author{
Abner Ramirez, Member, IEEE, Adam Semlyen, Life Fellow, IEEE, and Reza Iravani, Fellow, IEEE
}

\begin{abstract}
It is known that an untransposed open-ended line causes a negative sequence component in the current and the generator reacts through third and higher harmonic voltages. The currents and voltages can be greatly amplified by resonance to any of these harmonics. The phenomenon of machine-line interaction has traditionally been explained as a sequence of interactive events between the line and the machine. This paper presents a unified noniterative mathematical representation of this interaction. The analysis is formulated in the Harmonic Domain $a b c$-frame to detect resonance conditions and for the assessment of their stability.
\end{abstract}

Index Terms-Harmonic domain, harmonics, resonance, standing waves, state space methods, time-periodic systems, time-varying systems, unbalanced transmission line.

\section{INTRODUCTION}

$\mathbf{T}$ HE interaction of inductive and capacitive elements in a network can produce resonance effects. This can lead to overvoltages and failure in the electrical equipment. In addition, instability conditions could appear. Only a few research studies have presented numerical results on resonance conditions when a generator is connected to a capacitive load or a transmission line [1]-[4]. These reports explain the phenomenon as a sequence of effects without a comprehensive mathematical formulation. To fill the void, this paper presents an analytical methodology to characterize the interaction of such systems with the purpose of detecting resonance and instability conditions.

The phenomenon analyzed is related to any of the natural frequencies corresponding to a standing wave pattern on the open-ended transmission line. This may coincide with the fundamental or an odd harmonic of the power frequency. The source of the resonance is the positive-sequence, fundamental-frequency (Thevenin) machine voltage, and possibly some space harmonics due to saliency. If the line is unbalanced, the charging current will have a negative-sequence component which produces induced voltages in the machine of higher harmonic frequency. The interaction is complex and therefore an appropriate tool is needed for its quantitative description.

We use Harmonic Domain (HD) representation of the system to analyze its dynamic and steady state behavior. It allows us to obtain the resonance conditions for the system based on the eigenanalysis of its Harmonic Domain Transfer Function

Manuscript received November 20, 2003. This work was supported in part by the Natural Sciences and Engineering Research Council of Canada. Paper no. TPWRD-00589-2003.

A. Ramirez is with CINVESTAV-Guadalajara, Guadalajara, Jalisco, Mexico 45090 (e-mail: abner.ramirez@cts-design.com).

A. Semlyen and R. Iravani are with the Department of Electrical and Computer Engineering, University of Toronto, Toronto, ON M5S 3G4, Canada (e-mail: adam.semlyen@utoronto.ca; iravani@ecf.utoronto.ca.).

Digital Object Identifier 10.1109/TPWRD.2004.834678
(HDTF) [3], [5]. Taking into account that harmonics due to phase imbalance of the transmission line are an essential part of the analysis, we have chosen the $a b c$-frame of reference for the representation of the generator.

The paper is organized as follows. In Section II the general theory of Dynamic Harmonic Domain (DHD) is developed. The DHD models for the generator and the transmission line are presented in Section III. The interactions in steady and dynamic states are presented in Sections IV and V.

\section{General THEORY}

\section{A. Dynamic and Steady States in the Harmonic Domain}

A linear time periodic (LTP) system (e.g., the synchronous machine in $a b c$-frame in this study) can be transformed into a linear time invariant (LTI) system by using the extended or dynamic harmonic domain (DHD) representation [6]. One of the major advantages of the DHD is that the time periodic coefficient matrices become constant Toeplitz-type matrices. Consider for instance the LTP system for the scalar case

$$
\begin{aligned}
& \dot{x}=a_{p} x+b_{p} u \\
& y=c_{p} x+d_{p} u
\end{aligned}
$$

where subscript $p$ stands for time-periodic; for example, coefficient $a_{p}$ is defined as

$$
a_{p}=a_{-h} e^{-j h \omega_{o} t}+\cdots+a_{o}+\cdots+a_{h} e^{j h \omega_{o} t} .
$$

The state representation (1) can be expressed in DHD as [5], [6]

$$
\begin{aligned}
& \dot{x}=(A-S) x+B u \\
& y=C x+D u
\end{aligned}
$$

where the variables are now complex vectors with time-varying coefficients, e.g.,

$$
x=\left[\begin{array}{lllll}
x_{-h}(t) & \cdots & x_{o}(t) & \cdots & x_{h}(t)
\end{array}\right]^{T}
$$

where $T$ denotes transposed; matrix $A$ (and $B, C, D$ ) has the Toeplitz form

$$
A=\left[\begin{array}{ccccc}
a_{o} & a_{-1} & \cdots & a_{-h} & \\
a_{1} & & & & a_{-h} \\
\vdots & \ddots & \ddots & \ddots & \vdots \\
a_{h} & & & & a_{-1} \\
& a_{h} & \cdots & a_{1} & a_{o}
\end{array}\right]
$$


and $S$ is the operational matrix of differentiation

$$
S=\operatorname{diag}\left\{-j h \omega_{o}, \ldots,-j \omega_{o}, 0, j \omega_{o}, \ldots, j h \omega_{o}\right\} .
$$

In the three-phase case the scalar coefficients in (1) become matrices; each time-varying element in ( $3 a$ ) is formed by three elements; an element from ( $3 b$ ) becomes a Toeplitz submatrix, and each differentiator from (3c) is repeated three times.

The Harmonic Domain Dynamic Transfer Function (HDDTF) of (2) is [5]

$$
H(s)=C(s I+S-A)^{-1} B+D
$$

where $s$ is the complex frequency. If the variation with respect to time is suppressed in (2), then we have the steady state representation of the LTP system given by

$$
\begin{aligned}
& x=(S-A)^{-1} B u \\
& y=C x+D u
\end{aligned}
$$

and (4) becomes the steady state HDTF

$$
H(0)=C(S-A)^{-1} B+D .
$$

We have shown above that a time periodic phenomenon can be represented by the set of linear differential equations (2), with corresponding HDDTF as in (4). A particular case is the steady state in which (2a) is reduced to the set of algebraic equations (5a) by setting $\dot{x}=0$ and the corresponding HDTF (6) is obtained by setting $s=0$ in (4).

In this paper we focus primarily on the calculation of resonances of the LTP system in steady state by analyzing the HDTF from (6). In this case, the output (voltages and currents) is calculated directly from (5b). We are then also interested in assessing stability properties of the LTP system (1). This is done in the DHD via the eigenanalysis of the constant matrix $(A-S)$ in (2a). If in addition we are interested in calculating a transient, then the LTP model (1) or the LTI model (2) can be used. However, we notice that the latter is a higher-dimensional set of differential equations, according to the number of harmonics included, and therefore its computational efficiency is questionable.

\section{B. Harmonic Vector (HV) and Harmonic Domain Transfer Function (HDTF)}

Let us define vector $u$ (and $y$ ) as a Harmonic Vector (HV) with the property [7]

$$
u=\left[\begin{array}{c}
u_{\mathrm{up}} \\
u_{\text {down }}
\end{array}\right]
$$

where $u_{\text {down }}$ is the conjugate of $u_{\text {up }}$. We note that this is an unconventional way of defining $u_{\text {down }}$, the conventional definition of $u_{\text {down }}$ being the flipped conjugate of $u_{\text {up }}$ which would entail a permutation (flipping) of the elements of the matrices obtained below.
The Harmonic Domain Transfer Function (HDTF), $H$, is for a given harmonic ( $*$ denoting the conjugate) of the form

$$
H=\left[\begin{array}{cc}
P & Q \\
Q^{*} & P^{*}
\end{array}\right]
$$

which can be easily verified if we substitute (7) and (8) into the expression $y=H u$ and compare the resultant upper equation with the conjugate of the resultant lower equation.

In the following we show the relation between two eigenvectors, $e_{1}$ and $e_{2}$, of $H$ associated with a pair of complex-conjugate eigenvalues. Let the first eigenvector be partitioned into its upper and lower halves

$$
e_{1}=\left[\begin{array}{l}
v_{1} \\
v_{2}
\end{array}\right]
$$

Then we show that

$$
e_{2}=\left[\begin{array}{l}
v_{2}^{*} \\
v_{1}^{*}
\end{array}\right]
$$

Indeed, taking (8) into account, the equation $H e_{1}=\lambda e_{1}$ becomes

$$
\left[\begin{array}{cc}
P & Q \\
Q^{*} & P^{*}
\end{array}\right]\left[\begin{array}{l}
v_{1} \\
v_{2}
\end{array}\right]=\lambda\left[\begin{array}{l}
v_{1} \\
v_{2}
\end{array}\right]
$$

while $H e_{2}=\lambda^{*} e_{2}$ gives

$$
\left[\begin{array}{cc}
P & Q \\
Q^{*} & P^{*}
\end{array}\right]\left[\begin{array}{c}
v_{2}^{*} \\
v_{1}^{*}
\end{array}\right]=\lambda^{*}\left[\begin{array}{c}
v_{2}^{*} \\
v_{1}^{*}
\end{array}\right] \text {. }
$$

If we now take the conjugate of (10b), we find that it becomes (10a). Thus, $e_{1}$ and $e_{2}$ form a $\mathrm{HV}$ when combined as a pair with complex-conjugate coefficients. Similarly, an eigenvector corresponding to a real eigenvalue of $H$ is a HV by itself; this can be shown by assuming $\lambda$ a real number in the procedure above.

\section{TRANSMISSION LINE AND GENERATOR MODELS IN THE DHD}

\section{A. Transmission Line Model in Open Circuit}

The Frequency Domain current/voltage relation from the sending end ( $s$ also designates the stator terminal of the generator) for an open-ended transmission line is

$$
i_{s}=-Y_{c} \tanh (\gamma l) v_{s}
$$

where $Y_{c}$ and $\gamma$ represent the characteristic admittance and the propagation function, respectively, of a line of length $l\left(i_{s}\right.$ being the current entering the generator). Approximating the transfer function in (11) by rational functions yields, with conventional notations

$$
\begin{aligned}
\dot{x} & =A x+B v_{s} \\
i_{s} & =C x+D v_{s}
\end{aligned}
$$


where the poles of the approximation are contained in the diagonal matrix $A ; B$ is a column vector with entries normalized to "ones" and the residues of the realization are contained in $C$ [8]. The time domain representation (12) of the line can be expressed in the DHD as in (2). In Appendix A we present the derivation of (2) for the single-phase case; a similar procedure applies for the three-phase case.

\section{B. Generator Model}

As mentioned earlier, the generator is modeled in the $a b c$ frame. This allows us to include space harmonics in the inductive circuit (see Appendix B). In addition, in this frame the physical meaning of harmonics is clearer than in the $d q$-frame. The detailed model of the synchronous machine for time domain and DHD is presented in Appendix B, using the motor convention. A step-up transformer may be taken into account by lumping its inductances with those of the machine. For convenience we review in this section the main equations. These are, the time domain representation

$$
\begin{aligned}
\frac{d}{d t}\left[\begin{array}{l}
i_{s} \\
i_{r}
\end{array}\right]= & -\left[\begin{array}{ll}
L_{s s} & L_{s r} \\
L_{s r}^{T} & L_{r r}
\end{array}\right]^{-1}\left\{\left[\begin{array}{ll}
R_{s} & \\
& R_{r}
\end{array}\right]\right. \\
& \left.+\frac{d}{d t}\left[\begin{array}{ll}
L_{s s} & L_{s r} \\
L_{s r}^{T} & L_{r r}
\end{array}\right]\right\}\left[\begin{array}{l}
i_{s} \\
i_{r}
\end{array}\right]+\left[\begin{array}{ll}
L_{s s} & L_{s r} \\
L_{s r}^{T} & L_{r r}
\end{array}\right]^{-1}\left[\begin{array}{l}
v_{s} \\
v_{r}
\end{array}\right]
\end{aligned}
$$

and the DHD representation

$$
\begin{aligned}
{\left[\begin{array}{l}
\dot{i}_{s} \\
\dot{i}_{r}
\end{array}\right]=- } & {\left[\begin{array}{ll}
L_{s s} & L_{s r} \\
L_{s r}^{T} & L_{r r}
\end{array}\right]^{-1}\left\{\left[\begin{array}{ll}
R_{s} & \\
& R_{r}
\end{array}\right]+\left[\begin{array}{ll}
S_{s} & \\
& S_{r}
\end{array}\right]\right.} \\
& \left.\times\left[\begin{array}{ll}
L_{s s} & L_{s r} \\
L_{s r}^{T} & L_{r r}
\end{array}\right]\right\}\left[\begin{array}{l}
i_{s} \\
i_{r}
\end{array}\right]+\left[\begin{array}{ll}
L_{s s} & L_{s r} \\
L_{s r}^{T} & L_{r r}
\end{array}\right]^{-1}\left[\begin{array}{l}
v_{s} \\
v_{r}
\end{array}\right] .
\end{aligned}
$$

In the steady state (13b) becomes

$$
\left[\begin{array}{l}
v_{s} \\
v_{r}
\end{array}\right]=\left\{\left[\begin{array}{ll}
R_{s} & \\
& R_{r}
\end{array}\right]+\left[\begin{array}{ll}
S_{s} & \\
& S_{r}
\end{array}\right]\left[\begin{array}{ll}
L_{s s} & L_{s r} \\
L_{s r}^{T} & L_{r r}
\end{array}\right]\right\}\left[\begin{array}{l}
i_{s} \\
i_{r}
\end{array}\right] .
$$

\section{STEADY-STATE INTERACTION}

In this section we present the steady state analysis of a generator connected either to a capacitive load or to a transmission line. Although the first is not a practical system, it is used here to introduce the problem and to simplify the analysis of resonance conditions.

\section{A. Mathematical Description}

From here on, the field voltage of the generator is assumed a constant quantity and up to the \pm 8 and \pm 9 harmonics are taken into account for voltages and currents in the rotor and in the stator, respectively. For example, in the case of the generator model described in Appendix B, the vector $v_{r}$ of size $36 \times 1$ is formed by zeros except for the 17th element corresponding to the constant voltage of the field winding.
The synchronous machine represented by (13c) can be expressed as

$$
\left[\begin{array}{l}
v_{s} \\
v_{r}
\end{array}\right]=\left[\begin{array}{ll}
Z_{s s} & Z_{s r} \\
Z_{r s} & Z_{r r}
\end{array}\right]\left[\begin{array}{l}
i_{s} \\
i_{r}
\end{array}\right]
$$

Substituting $i_{r}$ from the second equation in (14) into the first equation yields

where

$$
v_{s}=Z_{g} i_{s}+e
$$

$$
Z_{g}=Z_{s s}-Z_{s r} Z_{r r}^{-1} Z_{r s} \text { and } e=Z_{s r} Z_{r r}^{-1} v_{r} .
$$

In (16b), $v_{r}$ is referred to the stator. This results in a vector $e$ of size $30 \times 1$ with 6 nonzero elements (positions 13 to 18 ), corresponding to the fundamental in phases $a, b$, and $c$. According to (15), $e$ can be seen as a positive sequence open circuit voltage. On the other hand, the terminal equation is

$$
i_{s}=-Y_{\text {load }} v_{s}
$$

where $Y_{\text {load }}$ represents the capacitive load or the line admittance given by (11). Combining (15) and (17) we get

$$
v_{s}=\left(I+Z_{g} Y_{\mathrm{load}}\right)^{-1} e=H e
$$

where $I$ is the identity matrix. Modal decomposition of $H$ gives

$$
v_{s}=T \Lambda T^{-1} e=T \Lambda e_{m}
$$

where $e_{m}$ is the modal representation of $e$, and $\Lambda$ the corresponding diagonal matrix of eigenvalues of $H$. Equation (19a) can be expressed also as

$$
v_{s}=\sum_{i} t_{i} \lambda_{i} e_{m_{i}}
$$

where $t_{i}$ is the $i$-th column (normalized to "unity" by Matlab) of the eigenvector matrix $T$. From (19b) we notice the possibility of an amplification of the output voltage $v_{s}$ for each eigenvector $t_{i}$ by the respective product of $\lambda_{i}$ and $e_{m_{i}}$; the elements of the corresponding eigenvector $t_{i}$ contain the information on which harmonics are amplified by $\lambda_{i} e_{m_{i}}$.

\section{B. Generator/C-Load Without Space Harmonics}

As a numerical example, consider an unbalanced load where the value $c_{a}$ of the capacitance in phase $a$, is varied from 0.001 p.u. to 10 p.u. in 500 steps; the values for the other phases are $c_{b}=0.1 c_{a}$ and $c_{c}=0.05 c_{a}$. Based on (19b), the upper plot in Fig. 1 shows the magnitude of the eigenvalues $\lambda_{i}$, the middle plot the magnitude of the products $\lambda_{i} e_{m_{i}}$ (only those bigger than unity), and the lower plot the norm of $v_{a}$ obtained from (18). The horizontal axis in Fig. 1 represents $c_{a}$. As stated earlier, we note from Fig. 1 that a resonance condition does not depend only on the biggest eigenvalues but on their product with the modal components of $e_{m}$.

The harmonic components of $v_{a}$ are shown in Fig. 2 as a function of $c_{a}$. The arrow in this figure indicates the most visible resonance occurring at $c_{a}=0.55$ p.u. For this value of 

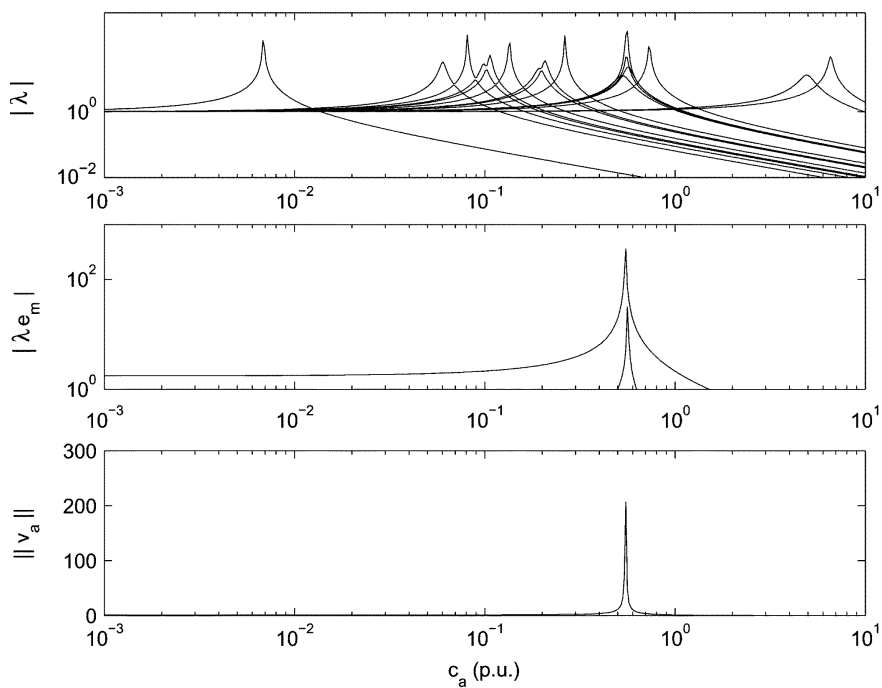

Fig. 1. Magnitude of eigenvalues $\lambda_{i}$, products $\lambda_{i} e_{m i}$, and norm of voltage $v_{a}$ without space harmonics.

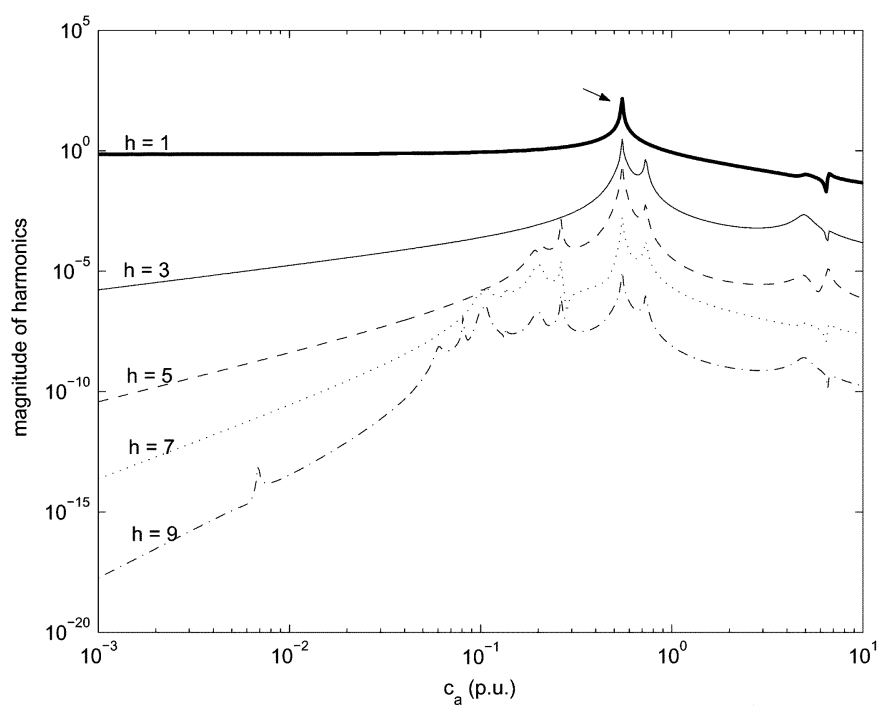

Fig. 2. Harmonic content of $v_{a}$, the arrow corresponds to $c_{a}=0.55$ p.u. without space harmonics.

capacitance the dominant harmonic corresponds to the fundamental as shown in Fig. 2. We can see clearly from this figure the frequency conversion effects, i.e., higher harmonics react to the negative sequence current generated by the unbalanced load. Similar results are obtained for terminal currents in the examples presented in this paper and, therefore, they are not shown.

Further experiments with more steps for $c_{a}$ show that the output voltages and currents can reach very big values at the resonance point limited only by the small amount of losses in the generator.

\section{Generator/C-Load With Space Harmonics}

Consider the system described above with the generator having space harmonics. In other words, the stator inductances have additionally even components up to the 10th harmonic and the stator-rotor mutual inductances contain odd components up to the 9th harmonic (see Appendix B). In this paper, we have considered $5,1,0.5$, and $0.1 \%$ of the 2 nd and of the
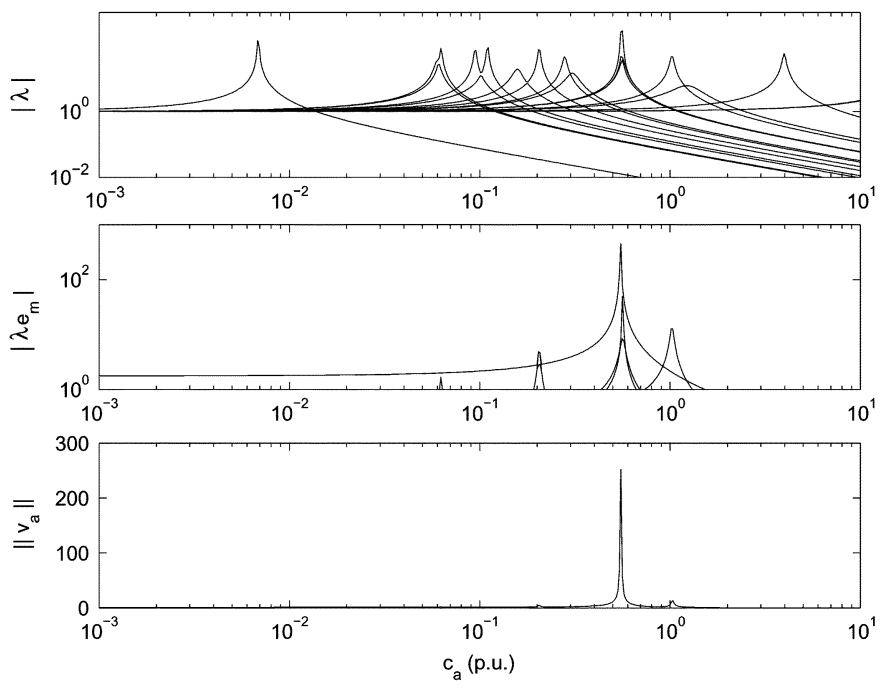

Fig. 3. Magnitude of eigenvalues $\lambda_{i}$, products $\lambda_{i} e_{m i}$, and norm of voltage $v_{a}$ with space harmonics.

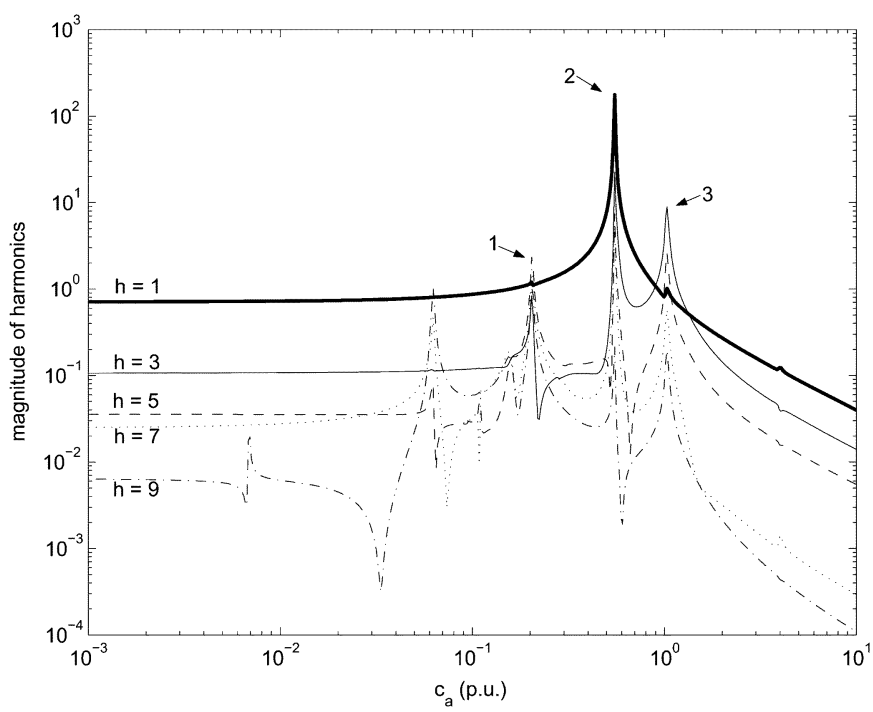

Fig. 4. Harmonic content of $v_{a}$, the arrows correspond to $c_{a}=0.2,0.55$, and 1.01 p.u. with space harmonics.

1st harmonic for stator and stator-rotor, respectively. A more detailed account on space harmonics can be found in [9].

Figs. 3 and 4 show results similar to those presented in Figs. 1 and 2. The most visible resonances appear at $c_{a}=0.2,0.55$, and 1.01 p.u. The harmonic content of $v_{a}$ for these resonance points is shown in Fig. 5 where the largest harmonic has been normalized to unity. From Figs. 4 and 5 we can see that the resonating harmonics of $v_{a}$ are the $5 \mathrm{th}, 1 \mathrm{st}$, and $3 \mathrm{rd}$. These figures show that higher harmonics are excited and produce resonances. This can be explained by vector $e$ being now a full $\mathrm{HV}$ as the impedance matrices in (16) include higher harmonic components due to space harmonics.

\section{Generator/Transmission Line Without Space Harmonics}

Next we analyze the case of a three-phase open-ended transmission line connected to a generator via an ideal transformer. As a numerical example, the length of a horizontal line, with height of $16 \mathrm{~m}$ and separation between conductors of $10 \mathrm{~m}$, has been varied from $10 \mathrm{~km}$ to $1000 \mathrm{~km}$ in 500 steps. Similarly to Figs. 1 and 2, the 


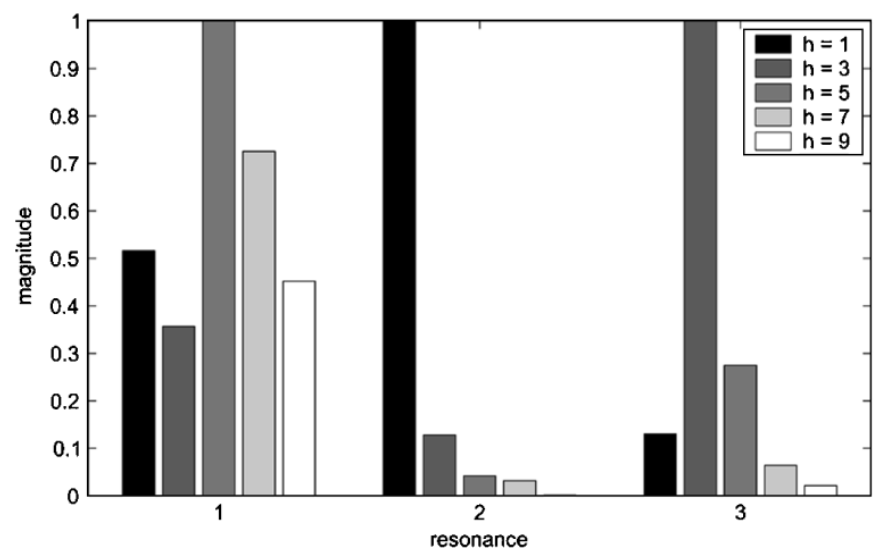

Fig. 5. Harmonic components of $v_{a}$ at $c_{a}=0.2,0.55$, and 1.01 p.u. with space harmonics.
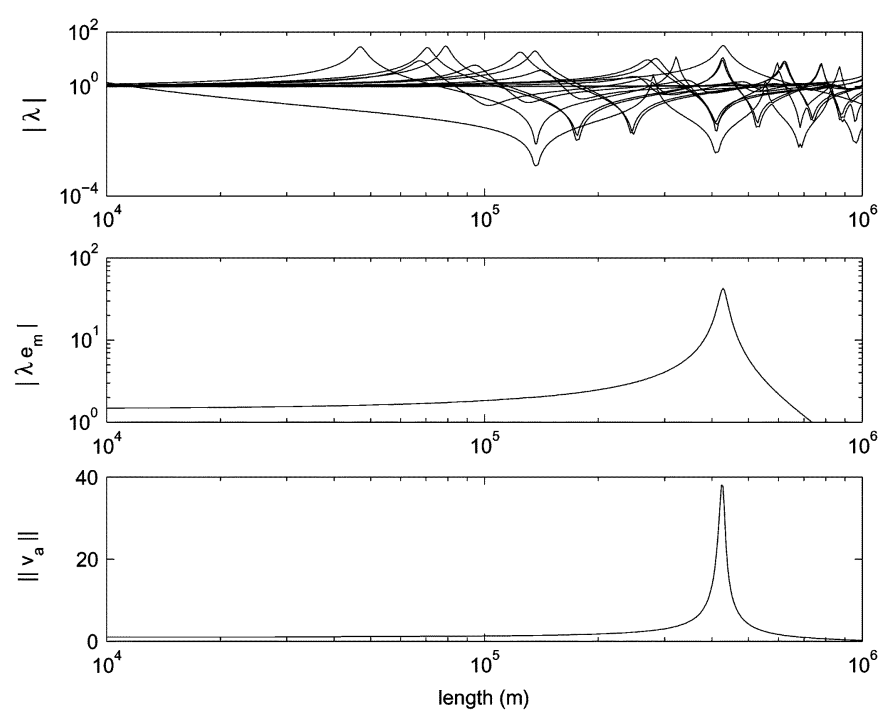

Fig. 6. Magnitude of eigenvalues $\lambda_{i}$, products $\lambda_{i} e_{m i}$, and norm of voltage $v_{a}$ without space harmonics.

results for this example are shown in Figs. 6 and 7 having now the length of the line in the horizontal axis. The point of resonance occurs at a length of $427.8 \mathrm{~km}$ with the fundamental dominant over higher harmonics as shown in Fig. 7.

In contrast to the capacitive load case, further experiments with finer discretization in the line lengths show that the magnitude of $v_{a}$ at the resonance point remains at the same value as in Fig. 6. This agrees with the fact that the system has higher losses.

\section{E. Generator/Transmission Line With Space Harmonics}

Consider next that the generator has space harmonics of the same magnitude as for the generator-capacitive load system of Section IV.C. Figs. 8 and 9 show results similar to those presented in Figs. 6 and 7. We note from these results that the additional resonances due to space harmonics are damped out in the case of a transmission line connected to the generator. The arrows in Fig. 9 correspond to resonances at $l=427.8$ and 618.8 $\mathrm{km}$. The harmonic content of $v_{a}$ for these resonance points is shown in Fig. 10 where the largest harmonic has been normalized to unity.

The two examples presented in this section correspond to slightly unbalanced systems. Further numerical experiments

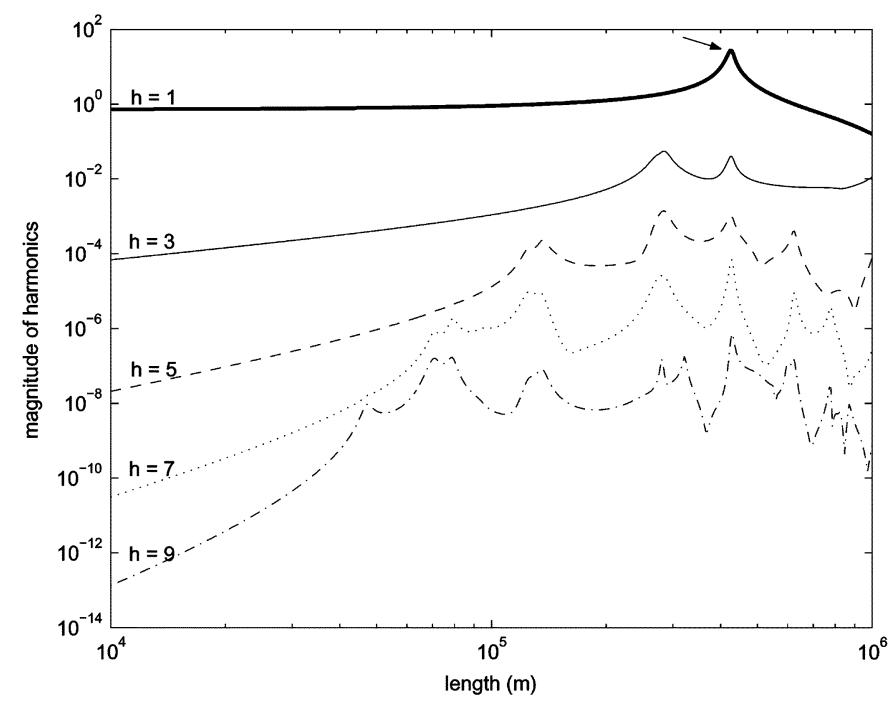

Fig. 7. Harmonic content of $v_{a}$, the arrow corresponds to $l=427.8 \mathrm{~km}$ without space harmonics.
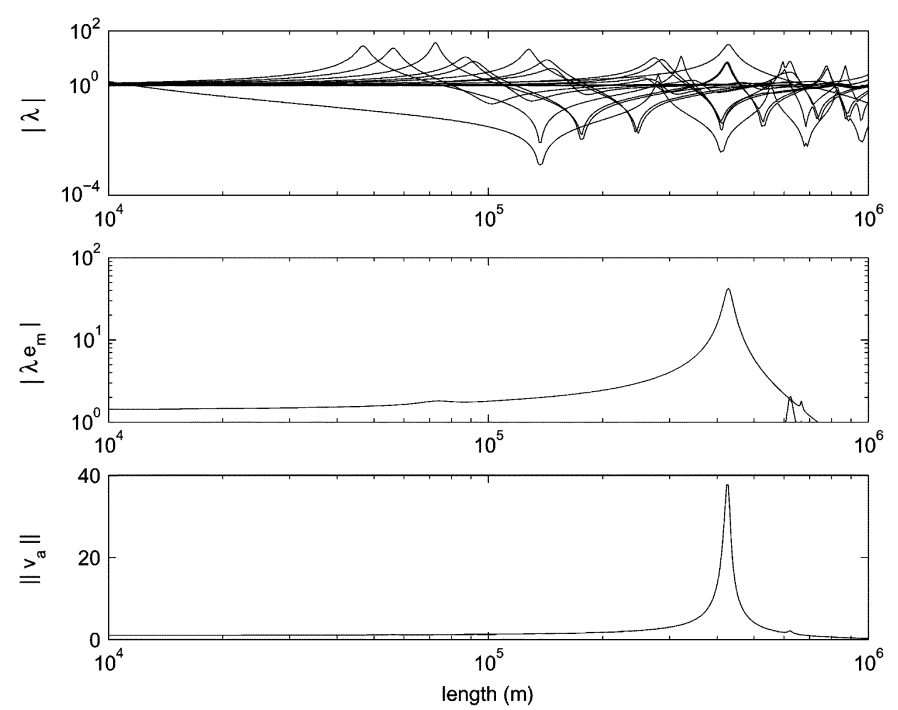

Fig. 8. Magnitude of eigenvalues $\lambda_{i}$, products $\lambda_{i} e_{m i}$, and norm of voltage $v_{a}$ with space harmonics.

with stronger unbalanced conditions have shown that the points of resonance are shifted according to the degree of unbalance. In addition, a corresponding change in the amplitude of the harmonic variables was observed.

\section{DYNAMIC INTERACTION}

In the present section, the systems of Section IV are formulated in the DHD and their stability properties are analyzed.

\section{A. Generator/C-Load}

The synchronous machine model in the DHD is expressed by (13b) and is repeated here as

$$
\left[\begin{array}{l}
\dot{i}_{s} \\
\dot{i}_{r}
\end{array}\right]=A_{g}\left[\begin{array}{c}
i_{s} \\
i_{r}
\end{array}\right]+L_{g}^{-1}\left[\begin{array}{l}
v_{s} \\
v_{r}
\end{array}\right] .
$$

Separating out the stator and rotor voltages in (20a) gives

$$
\left[\begin{array}{l}
\dot{i}_{s} \\
\dot{i}_{r}
\end{array}\right]=A_{g}\left[\begin{array}{l}
i_{s} \\
i_{r}
\end{array}\right]+L_{g 1}^{-1} v_{s}+L_{g 2}^{-1} v_{r} .
$$




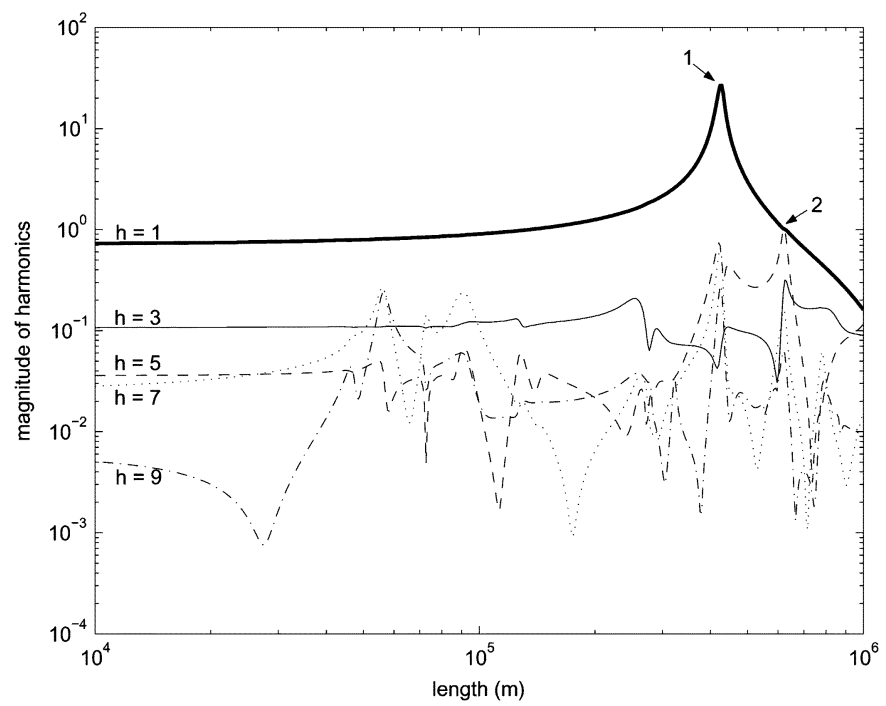

Fig. 9. Harmonic content of $v_{a}$, the arrow corresponds to $l=427.8$ and 618.8 $\mathrm{km}$ with space harmonics.

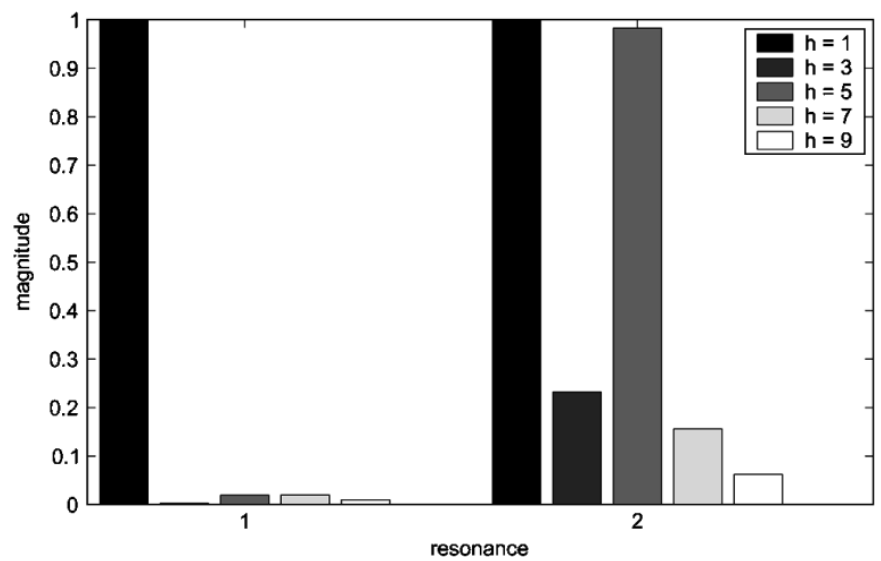

Fig. 10. Harmonic components of $v_{a}$ at $l=427.8$ and $618.8 \mathrm{~km}$ with space harmonics.

For the capacitive load the model is (currents entering the machine)

or

$$
\begin{aligned}
{\left[\begin{array}{c}
\dot{v}_{a} \\
\dot{v}_{b} \\
\dot{v}_{c}
\end{array}\right]=} & -\left[\begin{array}{lll}
S & & \\
& S & \\
& S
\end{array}\right]\left[\begin{array}{c}
v_{a} \\
v_{b} \\
v_{c}
\end{array}\right] \\
& -\left[\begin{array}{lll}
I / c_{a} & \\
& I / c_{b} & \\
& & I / c_{c}
\end{array}\right]\left[\begin{array}{c}
i_{a} \\
i_{b} \\
i_{c}
\end{array}\right]
\end{aligned}
$$

Equations (20b) and (21b) can be combined, yielding

$$
\begin{aligned}
& {\left[\begin{array}{l}
\dot{i}_{s} \\
\dot{i}_{r} \\
\dot{v}_{s}
\end{array}\right]=\left[\begin{array}{ccc}
A_{g} & L_{g 1}^{-1} \\
{\left[B_{c}\right.} & 0 & -A_{c}
\end{array}\right]\left[\begin{array}{c}
i_{s} \\
i_{r} \\
v_{s}
\end{array}\right]+\left[\begin{array}{c}
L_{g 2}^{-1} \\
0
\end{array}\right] v_{r}} \\
& {\left[\begin{array}{l}
i_{s} \\
v_{s}
\end{array}\right]=\left[\begin{array}{lll}
I & 0 & 0 \\
0 & 0 & I
\end{array}\right]\left[\begin{array}{l}
i_{s} \\
i_{r} \\
v_{s}
\end{array}\right]}
\end{aligned}
$$

or in a more compact form

$$
\begin{aligned}
& \dot{x}=A x+B v_{r} \\
& y=C x .
\end{aligned}
$$

From (23) we can obtain the input/output relation (similar to (4))

$$
y=H(s) v_{r}=C(s I-A)^{-1} B v_{r} .
$$

Equation (24) is equivalent to (18) when $s=0$.

From (24) we can see that the poles of $H(s)$ correspond to the eigenvalues of matrix $A$ in (22a). It is important to note that although the steady state of the system can always be computed from (18), its stability is defined by the eigenvalues of $A$ in (22a). This indicates that the steady state of the system can only be reached when it is stable.

The eigenvalues of $A$ from (22a) corresponding to the system generator/capacitive load (Section IV.B) were calculated for each value of capacitance. This resulted in a stable region for $c_{a}<0.55$ and unstable otherwise. Considering that the positive sequence inductance of the generator is 1.71 p.u., the natural frequency of oscillations at $c_{a}=0.6$ p.u. is $\omega_{n}=1 / \sqrt{(1.71)(0.6)}=0.98$ p.u. At this natural frequency the machine acts as an induction generator which feeds energy into the system. This explains the reason for the instability [10], [11].

From the results obtained in Section IV.B we notice that it is possible to reach the steady state for the resonant point at $c_{a}=0.55$ p.u. However, the eigenanalysis of (22a) leads to instability of the system. In fact, we did not take saturation in the generator into account and the losses are minimum, thus allowing for the flux to grow indefinitely. On the other hand, when a magnetic circuit saturates, the inductance decreases. If we consider this in the system of Section IV.B, the point at $c_{a}=0.55$ p.u. becomes stable. Therefore, this resonance is initially unstable but a stable solution may be reached if we let the inductance saturate or include enough losses in the system. In this paper the machine inductance matrix was reduced in a simple way by a factor $0<k<1$; a more complex representation of saturation [10]-[12] is not considered in this paper.

\section{B. Generator/Transmission Line}

From the DHD representation (30) of the transmission line (see Appendix A) we have

$$
v_{s}=D_{l}^{-1}\left(i_{s}-C_{l} x\right) .
$$

Substituting (25) into (30a) and (20b) gives

$$
\begin{aligned}
& \qquad \dot{x}=\left(A_{l}-S-B_{l} D_{l}^{-1} C_{l}\right) x+\left[\begin{array}{ll}
B_{l} D_{l}^{-1} & 0
\end{array}\right]\left[\begin{array}{l}
i_{s} \\
i_{r}
\end{array}\right] . \\
& \text { and } \\
& \qquad\left[\begin{array}{c}
\dot{i}_{s} \\
\dot{i}_{r}
\end{array}\right]=A_{g}^{\prime}\left[\begin{array}{c}
i_{s} \\
i_{r}
\end{array}\right]-L_{g 1}^{-1} D_{l}^{-1} C_{l} x+L_{g 2}^{-1} v_{r}
\end{aligned}
$$

where

$$
\begin{aligned}
A_{g}^{\prime}=-\left[\begin{array}{cc}
L_{s} & L_{s r} \\
L_{s r}^{T} & L_{r}
\end{array}\right]^{-1}\left\{\left[\begin{array}{cc}
R_{s}+D_{l}^{-1} & \\
& R_{r}
\end{array}\right]\right. \\
\left.+\left[\begin{array}{ll}
S_{s} & \\
& S_{r}
\end{array}\right]\left[\begin{array}{cc}
L_{s} & L_{s r} \\
L_{s r}^{T} & L_{r}
\end{array}\right]\right\} .
\end{aligned}
$$


Equations (25)-(27) are combined into

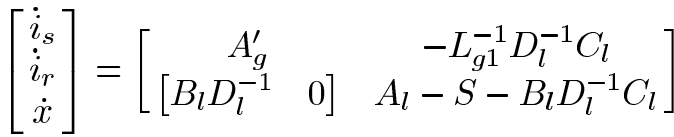

$$
\begin{aligned}
& \times\left[\begin{array}{c}
i_{s} \\
i_{r} \\
x
\end{array}\right]+\left[\begin{array}{c}
L_{g 2}^{-1} \\
0
\end{array}\right] v_{r} \\
& {\left[\begin{array}{c}
i_{s} \\
v_{s}
\end{array}\right]=\left[\begin{array}{ccc}
I & 0 & 0 \\
D_{l}^{-1} & 0 & -D_{l}^{-1} C_{l}
\end{array}\right]\left[\begin{array}{c}
i_{s} \\
i_{r} \\
x
\end{array}\right] \text {. }}
\end{aligned}
$$

Thus, the DHD system (28) can be expressed as in (23) or (24).

The eigenvalues of $A$ from (28a), corresponding to the system in Section IV.D, were calculated for line lengths up to $500 \mathrm{~km}$. In contrast to the pure capacitive load, all cases exhibit stable eigenvalues. Therefore, with this system we can obtain accurately by (28) via numerical integration the steady state values shown in the lowest plot of Fig. 6.

\section{Effect of Space Harmonics in the DHD}

We notice that space harmonics have not been included in the examples presented in the preceding Sections V-A and B. Numerical experiments have shown that the stability of the system is greatly affected by space harmonics. For example, stability is not assured for all the values of capacitance or lengths of line for the examples presented if $10 \%$ or more of the basic inductance terms is used to include space harmonics. We do not however pursue in detail in this paper the mathematical analysis of stability in the presence of space harmonics.

\section{Accuracy Assessment of Stability Analysis}

In this paper the stability of the system is assessed via the eigenanalysis of the HD matrix $A$ in (23a). A more accurate approach could be the application of Floquet theory [13] to the original LTP system (1). It implies examining the eigenvalues of the "average" of matrix $A_{p}$. Since the HD approach is obtained from the LTP system (1), both methods of assessing stability become equivalent if enough harmonics are included in the former, i.e., if the truncation error is minimized.

\section{CONCLUSION}

The interaction between a system consisting of a generator and a capacitive load or an open-ended transmission line is analyzed. The modeling of these components is done in the Harmonic Domain $a b c$-frame of reference, making possible the inclusion of imbalances and shedding light on frequency conversion effects. Resonance conditions were characterized using the static model of the system while stability issues have been addressed using its dynamic formulation.

Since the transmission line has several natural frequencies, more than one pattern of resonance may exist. In some of these the fundamental is not necessarily dominant. The possibility of dominance for higher harmonics become more pronounced in the presence of space harmonics in the generator.
An important result is also that the system can be unstable if the element connected to the generator does not have enough losses or if saturation is not included in the modeling of the generator.

\section{APPENDIX A}

\section{LINE MODEL IN THE DHD}

For simplicity, consider that only two poles are used for the rational approximation [8] of the transfer function (11). Then (12) becomes

$$
\begin{aligned}
{\left[\begin{array}{l}
\dot{x}_{1} \\
\dot{x}_{2}
\end{array}\right] } & =\left[\begin{array}{ll}
a_{1} & \\
& a_{2}
\end{array}\right]\left[\begin{array}{l}
x_{1} \\
x_{2}
\end{array}\right]+\left[\begin{array}{l}
1 \\
1
\end{array}\right] v_{s} \\
i_{s} & =\left[\begin{array}{ll}
c_{1} & c_{2}
\end{array}\right]\left[\begin{array}{l}
x_{1} \\
x_{2}
\end{array}\right]+d v_{s} .
\end{aligned}
$$

System (29) can be expressed in the DHD, similarly to (2), as

$$
\begin{aligned}
\dot{x} & =\left(A_{l}-S\right) x+B_{l} v_{s} \\
i_{s} & =C_{l} x+D_{l} v_{s} .
\end{aligned}
$$

Considering up to harmonic \pm 3 , the vectors and matrices in (30) have the following structure

$$
\begin{aligned}
& x=\left[\begin{array}{llllllll}
x_{1}^{-3} & x_{1}^{-1} & x_{1}^{+1} & x_{1}^{+3} & x_{2}^{-3} & x_{2}^{-1} & x_{2}^{+1} & x_{2}^{+3}
\end{array}\right]^{T} \\
& v_{s}=\left[\begin{array}{llll}
v^{-3} & v^{-1} & v^{+1} & v^{+3}
\end{array}\right]^{T} \\
& i_{s}=\left[\begin{array}{llll}
i^{-3} & i^{-1} & i^{+1} & i^{+3}
\end{array}\right]^{T} \\
& S=\operatorname{diag}\left\{S_{1}, S_{1}\right\}
\end{aligned}
$$

where

$$
\begin{aligned}
& S_{1}=\operatorname{diag}\left\{-j 3 \omega_{o},-j \omega_{o}, j \omega_{o}, j 3 \omega_{o}\right\} \\
& A_{l}=\operatorname{diag}\left\{a_{1} I_{4}, a_{2} I_{4}\right\}, \quad B_{l}=\left[\begin{array}{c}
I_{4} \\
I_{4}
\end{array}\right] \\
& C_{l}=\left[\begin{array}{ll}
c_{1} I_{4} & c_{2} I_{4}
\end{array}\right], \quad D_{l}=d I_{4}
\end{aligned}
$$

with $I_{4}$ the identity matrix of size 4 .

\section{APPENDIX B \\ Generator MODEL IN THE DHD}

We have adopted the notation from [11] for the representation of a generator (in this paper we use the terms generator and synchronous machine interchangeably) with two windings along the $d$-axis ( $F$ and $D)$ and two along the $q$-axis ( $G$ and $Q)$. Although any number of space harmonics can be considered, for simplicity only up to the second harmonic is included in the derivation below.

\section{A. Basic Parameters}

The data for the generator used in this paper have been adopted from [10] but ensuring the positive definiteness of the inductance matrix. The following definition is used:

$$
\theta=\omega_{o} t+\theta_{o}
$$

where the rotor speed $\omega_{r}$ has been taken equal to the system base frequency $\omega_{o}$. The angle $\theta_{0}$ is considered for a constant 
load angle and can be calculated by traditional procedures as in [10].

i) Stator Self and Mutual Inductances

$$
\begin{aligned}
& L_{a a}=L_{s}+L_{m} \cos 2 \theta \\
& L_{a b}=-M_{s}-L_{m} \cos 2(\theta+\pi / 6) \\
& L_{b b}=L_{s}+L_{m} \cos 2(\theta-2 \pi / 3) \\
& L_{b c}=-M_{s}-L_{m} \cos 2(\theta-\pi / 2) \\
& L_{c c}=L_{s}+L_{m} \cos 2(\theta+2 \pi / 3) \\
& L_{c a}=-M_{s}-L_{m} \cos 2(\theta+5 \pi / 6) .
\end{aligned}
$$

ii) Rotor Self Inductances

$$
L_{F}, L_{D}, L_{G}, \text { and } L_{Q} .
$$

iii) Rotor Mutual Inductances

$$
\begin{aligned}
& L_{F D}=M_{X}, \quad L_{G Q}=M_{Y} \\
& L_{F G}=L_{F Q}=L_{D G}=L_{D Q}=0 .
\end{aligned}
$$

iv) Stator-Rotor Mutual Inductances

$$
\begin{array}{ll}
L_{a F}=M_{F} \cos \theta, & L_{a D}=M_{D} \cos \theta \\
L_{b F}=M_{F} \cos (\theta-2 \pi / 3), & L_{b D}=M_{D} \cos (\theta-2 \pi / 3) \\
L_{c F}=M_{F} \cos (\theta+2 \pi / 3) & L_{c D}=M_{D} \cos (\theta+2 \pi / 3) \\
L_{a G}=M_{G} \sin \theta, & L_{a Q}=M_{Q} \sin \theta \\
L_{b G}=M_{G} \sin (\theta-2 \pi / 3), & L_{b Q}=M_{Q} \sin (\theta-2 \pi / 3) \\
L_{c G}=M_{G} \sin (\theta+2 \pi / 3), & L_{c Q}=M_{Q} \sin (\theta+2 \pi / 3) .
\end{array}
$$

If space harmonics are considered for the machine model, then the inductances from $i$ ) and $i v$ ) should be modified. Two examples are

$$
\begin{aligned}
L_{b b} & =L_{s}+\sum_{h} L_{m_{h}} \cos h(\theta-2 \pi / 3), \quad h=2,4, \ldots \\
L_{c D} & =\sum_{h} M_{D_{h}} \cos h(\theta+2 \pi / 3), \quad h=1,3, \ldots
\end{aligned}
$$

\section{B. Time Domain Representation}

The flux linkages are related with the currents by

$$
\begin{aligned}
{\left[\begin{array}{l}
\psi_{a} \\
\psi_{b} \\
\psi_{c} \\
\psi_{F} \\
\psi_{D} \\
\psi_{G} \\
\psi_{Q}
\end{array}\right]=} & {\left[\begin{array}{lllllll}
L_{a a} & L_{a b} & L_{a c} & L_{a F} & L_{a D} & L_{a G} & L_{a Q} \\
L_{b a} & L_{b b} & L_{b c} & L_{b F} & L_{b D} & L_{b G} & L_{b Q} \\
L_{c a} & L_{c b} & L_{c c} & L_{c F} & L_{c D} & L_{c G} & L_{c Q} \\
L_{F a} & L_{F b} & L_{F c} & L_{F} & M_{X} & & \\
L_{D a} & L_{D b} & L_{D c} & M_{X} & L_{D} & & \\
L_{G a} & L_{G b} & L_{G c} & & & L_{G} & M_{Y} \\
L_{Q a} & L_{Q b} & L_{Q c} & & & M_{Y} & L_{Q}
\end{array}\right] } \\
& \times\left[\begin{array}{c}
i_{a} \\
i_{b} \\
i_{c} \\
i_{F} \\
i_{D} \\
i_{G} \\
i_{Q}
\end{array}\right]
\end{aligned}
$$

or

$$
\left[\begin{array}{l}
\psi_{s} \\
\psi_{r}
\end{array}\right]=\left[\begin{array}{ll}
L_{s s} & L_{s r} \\
L_{s r}^{T} & L_{r r}
\end{array}\right]\left[\begin{array}{l}
i_{s} \\
i_{r}
\end{array}\right]
$$

Adopting the representation of the synchronous machine as a motor we have

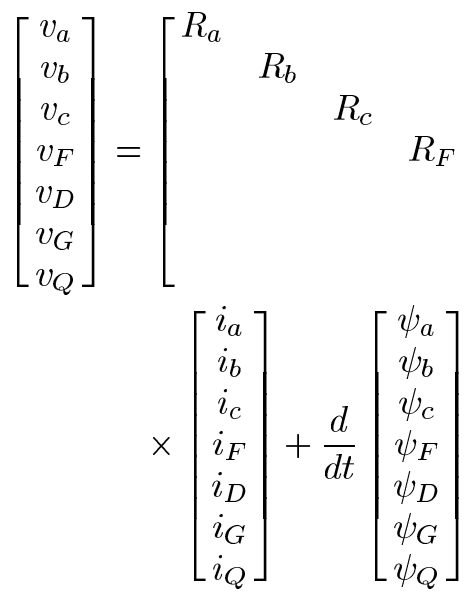

or

$$
\left[\begin{array}{l}
v_{s} \\
v_{r}
\end{array}\right]=\left[\begin{array}{ll}
R_{s} & \\
& R_{r}
\end{array}\right]\left[\begin{array}{l}
i_{s} \\
i_{r}
\end{array}\right]+\frac{d}{d t}\left[\begin{array}{l}
\psi_{s} \\
\psi_{r}
\end{array}\right]
$$

Evaluation of the derivative of the flux in (33b) yields

$$
\begin{aligned}
& {\left[\begin{array}{l}
v_{s} \\
v_{r}
\end{array}\right]=\left\{\left[\begin{array}{ll}
R_{s} & \\
& R_{r}
\end{array}\right]+\frac{d}{d t}\left[\begin{array}{ll}
L_{s s} & L_{s r} \\
L_{s r}^{T} & L_{r r}
\end{array}\right]\right\} } \\
& \times\left[\begin{array}{l}
i_{s} \\
i_{r}
\end{array}\right]+\left[\begin{array}{ll}
L_{s s} & L_{s r} \\
L_{s r}^{T} & L_{r r}
\end{array}\right] \frac{d}{d t}\left[\begin{array}{l}
i_{s} \\
i_{r}
\end{array}\right] .
\end{aligned}
$$

From (34) we get (13a).

\section{Dynamic Harmonic Domain Representation}

The representation of (34) in the DHD is

$$
\begin{aligned}
{\left[\begin{array}{l}
v_{s} \\
v_{r}
\end{array}\right]=\left\{\left[\begin{array}{ll}
R_{s s} & \\
& R_{r r}
\end{array}\right]\right.} & \left.+\left[\begin{array}{ll}
S_{s} & \\
& S_{r}
\end{array}\right]\left[\begin{array}{ll}
L_{s s} & L_{s r} \\
L_{s r}^{T} & L_{r r}
\end{array}\right]\right\} \\
& \times\left[\begin{array}{l}
i_{s} \\
i_{r}
\end{array}\right]+\left[\begin{array}{ll}
L_{s s} & L_{s r} \\
L_{s r}^{T} & L_{r r}
\end{array}\right]\left[\begin{array}{l}
\dot{i}_{s} \\
\dot{i}_{r}
\end{array}\right]
\end{aligned}
$$

which becomes (13b).

For simplicity of illustration, the highest harmonic for stator variables is equal to \pm 3 and for rotor variables is equal to \pm 2 . Therefore, we have the following vectors and matrices corresponding to (35), shown in the equations at the top of the page.

The last element of (35) to be described is the total inductance matrix. To illustrate its structure, we take only four representative inductance terms from (32a) as described below.

i) Stator self-inductance

Consider the self-inductance of phase $a$

$$
\begin{aligned}
L_{a a} & =L_{s}+L_{m} \cos 2\left(\omega_{o} t+\theta_{o}\right) \\
& =L_{s}+\frac{L_{m}}{2} e^{-j 2 \theta_{o}} e^{-j 2 \omega_{o} t}+\frac{L_{m}}{2} e^{j 2 \theta_{o}} e^{j 2 \omega_{o} t} \\
L_{a a} & =L_{a a}^{o}+L_{a a}^{-2} e^{-j 2 \omega_{o} t}+L_{a a}^{+2} e^{j 2 \omega_{o} t} .
\end{aligned}
$$$$
\text { or }
$$ 


$$
\begin{aligned}
& v_{s}=\left[\begin{array}{llllllllllll}
v_{a}^{-3} & v_{b}^{-3} & v_{c}^{-3} & v_{a}^{-1} & v_{b}^{-1} & v_{c}^{-1} & v_{a}^{+1} & v_{b}^{+1} & v_{c}^{+1} & v_{a}^{+3} & v_{b}^{+3} & v_{c}^{+3}
\end{array}\right]^{T} \\
& v_{r}=\left[\begin{array}{llllllllllll}
0 & 0 & 0 & 0 & v_{F}^{o} & 0 & 0 & 0 & 0 & 0 & 0 & 0
\end{array}\right]^{T} \\
& i_{s}=\left[\begin{array}{llllllllllll}
i_{a}^{-3} & i_{b}^{-3} & i_{c}^{-3} & i_{a}^{-1} & i_{b}^{-1} & i_{c}^{-1} & i_{a}^{+1} & i_{b}^{+1} & i_{c}^{+1} & i_{a}^{+3} & i_{b}^{+3} & i_{c}^{+3}
\end{array}\right]^{T} \\
& i_{r}=\left[\begin{array}{llllllllllll}
i_{F}^{-2} & i_{D}^{-2} & i_{G}^{-2} & i_{Q}^{-2} & i_{F}^{o} & i_{D}^{o} & i_{G}^{o} & i_{Q}^{o} & i_{F}^{+2} & i_{D}^{+2} & i_{G}^{+2} & i_{Q}^{+2}
\end{array}\right]^{T} \\
& S_{s}=\operatorname{diag}\left\{-j 3 \omega_{o} I_{3},-j \omega_{o} I_{3}, j \omega_{o} I_{3}, j 3 \omega_{o} I_{3}\right\} \\
& S_{r}=\operatorname{diag}\left\{-j 2 \omega_{o} I_{4}, 0,0,0,0, j 2 \omega_{o} I_{4}\right\} \\
& R_{s s}=\operatorname{diag}\left\{R_{a}, R_{b}, R_{c}, R_{a}, R_{b}, R_{c}, R_{a}, R_{b}, R_{c}, R_{a}, R_{b}, R_{c},\right\} \\
& R_{r r}=\operatorname{diag}\left\{R_{F}, R_{D}, R_{G}, R_{Q}, R_{F}, R_{D}, R_{G}, R_{Q}, R_{F}, R_{D}, R_{G}, R_{Q}\right\} .
\end{aligned}
$$

This inductance multiplies a stator current vector to produce a stator flux vector as follows:

$$
\left[\begin{array}{cccc}
L_{a a}^{o} & L_{a a}^{-2} & & \\
L_{a a}^{+2} & L_{a a}^{o} & L_{a a}^{-2} & \\
& L_{a a}^{+2} & L_{a a}^{o} & L_{a a}^{-2} \\
& & L_{a a}^{+2} & L_{a a}^{o}
\end{array}\right]\left[\begin{array}{c}
i_{a}^{-3} \\
i_{a}^{-1} \\
i_{a}^{+1} \\
i_{a}^{+3}
\end{array}\right]=\left[\begin{array}{c}
\psi_{a}^{-3} \\
\psi_{a}^{-1} \\
\psi_{a}^{+1} \\
\psi_{a}^{+3}
\end{array}\right] .
$$

ii) Stator-rotor inductance

We have the inductance between phase $a$ and the field winding

or

$$
\begin{aligned}
L_{a F} & =M_{F} \cos \left(\omega_{o} t+\theta_{o}\right) \\
& =\frac{M_{F}}{2} e^{-j \theta_{o}} e^{-j \omega_{o} t}+\frac{M_{F}}{2} e^{j \theta_{o}} e^{j \omega_{o} t}
\end{aligned}
$$

$$
L_{a F}=L_{a F}^{-1} e^{-j \omega_{o} t}+L_{a F}^{+1} e^{j \omega_{o} t} .
$$

This inductance multiplies a rotor current vector to produce a stator flux vector as follows:

$$
\left[\begin{array}{ccc}
L_{a F}^{-1} & & \\
L_{a F}^{+1} & L_{a F}^{-1} & \\
& L_{a F}^{+1} & L_{a F}^{-1} \\
& & L_{a F}^{+1}
\end{array}\right]\left[\begin{array}{c}
i_{F}^{-2} \\
i_{F}^{o} \\
i_{F}^{+2}
\end{array}\right]=\left[\begin{array}{c}
\psi_{a}^{-3} \\
\psi_{a}^{-1} \\
\psi_{a}^{+1} \\
\psi_{a}^{+3}
\end{array}\right] .
$$

iii) Rotor-stator inductance

In this case we have between the field winding and phase $a, L_{F a}=L_{a F}$. This inductance multiplies a stator current vector to produce a rotor flux vector as follows:

$$
\left[\begin{array}{cccc}
L_{a F}^{+1} & L_{a F}^{-1} & & \\
& L_{a F}^{+1} & L_{a F}^{-1} & \\
& & L_{a F}^{+1} & L_{a F}^{-1}
\end{array}\right]\left[\begin{array}{c}
i_{a}^{-3} \\
i_{a}^{-1} \\
i_{a}^{+1} \\
i_{a}^{+3}
\end{array}\right]=\left[\begin{array}{c}
\psi_{F}^{-2} \\
\psi_{F}^{o} \\
\psi_{F}^{+2}
\end{array}\right] .
$$

iv) Rotor self-inductance

Consider the field inductance, which multiplies a rotor current vector to produce a rotor flux vector as follows:

$$
\left[\begin{array}{lll}
L_{F} & & \\
& L_{F} & \\
& & L_{F}
\end{array}\right]\left[\begin{array}{c}
i_{F}^{-2} \\
i_{F}^{o} \\
i_{F}^{+2}
\end{array}\right]=\left[\begin{array}{c}
\psi_{F}^{-2} \\
\psi_{F}^{o} \\
\psi_{F}^{+2}
\end{array}\right] .
$$

All matrices in (36a)-(36d), and the ones obtained from similar inductance terms, are used to form the total inductance matrix in (35).

\section{REFERENCES}

[1] E. Acha and M. Madrigal, Power Systems Harmonics. New York: Wiley, 2001.

[2] A. Semlyen, J. F. Eggleston, and J. Arrillaga, "Admittance matrix model of a synchronous machine for harmonic analysis," IEEE Trans. Power Syst., vol. PS-2, no. 4, pp. 833-840, Nov. 1987.

[3] A. Semlyen, E. Acha, and J. Arrillaga, "Newton-type algorithms for the harmonic phasor analysis of nonlinear power circuits in periodical steady state with special reference to magnetic nonlinearities," IEEE Trans. Power Del., vol. 3, no. 3, pp. 1090-1098, Jul. 1988.

[4] W. W. Xu, H. W. Dommel, and J. R. Marti, "A synchronous machine model for three-phase harmonic analysis and EMTP initialization," IEEE Trans. Power Syst., vol. 6, no. 4, pp. 1530-1538, Nov. 1991.

[5] T. Noda, A. Semlyen, and R. Iravani, "Harmonic domain dynamic transfer function of a nonlinear time-periodic network," IEEE Trans. Power Del., vol. 18, no. 4, pp. 1433-1441, Oct. 2003.

[6] J. J. Rico, M. Madrigal, and E. Acha, "Dynamic harmonic evolution using the extended harmonic domain," IEEE Trans. Power Del., vol. 18, no. 2, pp. 587-594, Apr. 2003.

[7] A. Semlyen, "Steady-state analysis of nonlinear dynamic systems with periodic excitation based on linearization in harmonic space," Can. Elect. Eng. J., vol. 11, no. 3, pp. 114-117, 1986.

[8] B. Gustavsen, "Computer code for rational approximation of frequency dependent admittance matrices," IEEE Trans. Power Del., vol. 17, no. 4, pp. 1093-1098, Oct. 2002.

[9] F. P. de Mello, "Measurement of synchronous machine rotor angle from analysis of zero sequence harmonic components of machine terminal voltage," IEEE Trans. Power Del., vol. 9, no. 4, pp. 1770-1777, Oct. 1994.

[10] P. Kundur, Power System Stability and Control. New York: McGrawHill, 1994.

[11] P. M. Anderson, B. L. Agrawal, and J. E. Van Ness, Subsynchronous Resonance in Power Systems. New York: IEEE Press, 1990.

[12] J. R. Marti and K. W. Louie, "A phase-domain synchronous generator model including saturation effects," IEEE Trans. Power Syst., vol. 12, no. 1, pp. 222-229, Feb. 1997.

[13] H. D'Angelo, Linear Time-Varying Systems: Analysis and Synthesis. Boston, MA: Allyn \& Bacon, 1970.

Abner Ramirez (M'96) received the B.Sc. degree from the University of Guanajuato, Guanajuato, Mexico, in 1996, the M.Sc. degree from the University of Guadalajara, Guadalajara, Mexico, in 1998, and the Ph.D. degree from the Center for Research and Advanced Studies of Mexico (CINVESTAV), Campus, Guadalajara, in 2001.

Currently, he is a Professor with CINVESTAV-Guadalajara, Guadalajara, Mexico. His interests are electromagnetic transient analysis in power systems and numerical analysis of electromagnetic fields. 
Adam Semlyen (LF'97) was born in 1923 in Rumania. He received the Dipl. Ing. degree from the Polytechnic Institute of Timisoara, Rumania, in 1950, and the Ph.D. degree from the Polytechnic Institute of Iasi, Rumania, in 1965.

He began his career with an electric power utility in Timisoara, Rumania, and held academic positions at the Polytechnic Institute. In 1969, he joined the University of Toronto, ON, Canada, where he is currently a Professor in the Department of Electrical and Computer Engineering (Emeritus since 1988). His research interests include steady-state and dynamic analysis, as well as computation, of electromagnetic transients in power systems.
Reza Iravani (F'03) received the B.Sc. degree in electrical engineering from Tehran Polytechnique University, Tehran, Iran, in 1976, and the M.Sc. and Ph.D. degrees in electrical engineering from the University of Manitoba, Winnipeg, MB, Canada, in 1981 and 1985, respectively.

He began his career as a Consulting Engineer in Iran. Currently, he is a Professor at the University of Toronto. His research interests include power electronics and power system dynamics and control. 